\title{
A CHARACTERIZATION OF THE EUCLIDEAN SPHERE
}

\author{
BY R. L. BISHOP AND S. I. GOLDBERG ${ }^{1}$
}

Communicated by W. S. Massey, September 28, 1965

1. Introduction. Let $M$ be a connected Riemannian manifold of dimension $n, C_{0}(M)$ its largest connected group of conformal transformations and $I_{0}(M)$ its largest connected group of isometries. In an earlier paper [2], one of the authors and S. Kobayashi established the following result:

THEOREM 1. A compact homogeneous Riemannian manifold for which $C_{0}(M) \neq I_{0}(M)$ and $n>3$ is globally isometric with a sphere. ${ }^{2}$

In the final step of the proof of this theorem the following statement, which is by no means easy to establish, was utilized:

Proposition 1 (Yano-Nagano [6]). A complete Einstein space for which $C_{0}(M) \neq I_{0}(M)$ and $n>2$ is globally isometric with a sphere.

Without this fact it was shown that the simply connected Riemannian covering of $M$ is globally isometric with a sphere. Using this statement, an elementary proof of Theorem 1, i.e. a proof which does not use Proposition 1, is given (see Proposition 4).

All other results in this direction employ Proposition 1 in the final analysis. We list several of these:

Proposition 2 (Nagano [4]). A complete Riemannian manifold with parallel Ricci tensor for which $C_{0}(M) \neq I_{0}(M)$ and $n>2$ is globally isometric with a sphere.

This generalizes Proposition 1.

Proposition 3 (Lichnerowicz [3]). Let $M$ be a compact Riemannian manifold of dimension $n>2$ whose scalar curvature $R$ is a positive constant and for which trace $Q^{2}=$ const. where $Q$ is the Ricci operator (see $[1, p .87])$. Then, if $C_{0}(M) \neq I_{0}(M), M$ is globally isometric with a sphere.

This generalizes Theorem 1 and Proposition 2.

In $\$ 4$, Proposition 1 will be generalized. Denote the Lie algebra of

1 The research of both authors was supported by NSF Grant GP 3624 .

2 The first part of the proof of Theorem 1 appears in a previous paper published in the Amer. J. Math 84 (1962), 170-174 by S. I. Goldberg and S. Kobayashi entitled The conformal transformation group of a compact Riemannian manifold. 
$C_{0}(M)$ by $C_{0}(M)$. Let $X \in C_{0}(M)$ and $\xi$ be the covariant form of $X$ defined by duality by the Riemannian metric $\langle$,$\rangle of M: \xi=\langle X, \cdot\rangle$. Let $C_{0}^{*}(M)=\left\{\xi \mid \xi=\langle X, \cdot\rangle, X \in C_{0}(M)\right\}$ and denote by $d$ and $\delta$ the differential and codifferential operators of de Rham and Hodge. Then [cf. M. Obata and K. Yano, C. R. Acad. Sci. Paris 260 (1965), 2698$2700]$.

THEOREM 2. Let $M$ be a compact Riemannian manifold of dimension $n>3$ for which $R=$ const. and $C_{0}(M) \neq I_{0}(M)$. If $d \delta C_{0}^{*}(M)$ is an invariant subspace of $Q$, then $M$ is globally isometric with a sphere.

This theoremal most completely answers the question raised in [2], namely,

Is a compact manifold of dimension $n>2$ with constant (positive) scalar curvature for which $C_{0}(M) \neq I_{0}(M)$ isometric with a sphere?

Observe that Proposition 1 is an easy consequence of Theorem 2.

2. Isometries and conformal fields. If $T$ is an isometry of the unit sphere $S^{n}$ in $E^{n+1}$, then $T$ may be viewed as an orthogonal linear transformation of $E^{n+1}$ restricted to $S^{n}$. It is clear that any such isometry will map Killing fields into Killing fields and constant conformal fields $(d \phi=\langle X, \cdot\rangle)$ into constant conformal fields. Thus if a conformal field is invariant under $T$ so are its constant and Killing parts. It follows that if $T$ leaves a non-Killing conformal field invariant then it has a fixed point, namely $N /\|N\| \in S^{n}$, where $N$ is a constant field in $E^{n+1}$ and $N-\langle N, P\rangle P\left(P \in S^{n}\right)$ is the constant part of $V$.

3. Conformal fields on a manifold of positive constant curvature. If $M$ is a compact Riemannian manifold with constant positive curvature then the nature of the conformal group of $M$ does not change if we normalized the curvature so that it is 1 . Thus, $S^{n}$ is the simply connected covering Riemannian manifold of $M$. If $M$ has a non-Killing conformal vector field $V$ then this vector field may be lifted to a non-Killing conformal vector field $\bar{V}$ on $S^{n}$. Moreover, $\bar{V}$ is invariant by the deck transformations of the covering space $S^{n} \rightarrow M$. But only the identity deck transformation can have a fixed point, and since a deck transformation is an isometry we have from $\$ 2$ that there are no deck transformations except the identity. This proves the following special case of Proposition 1:

Proposition 4. If a compact Riemannian manifold of positive constant curvature admits a non-Killing conformal vector field then it is globally isometric with a sphere.

Since the above argument clearly works for $n=2$, we have 
COROLlARY. The real projective plane does not admit a non-Killing conformal vector field.

4. Conformal fields on manifolds of constant scalar curvature. We sketch the proof of Theorem 2. Let $\xi=d \phi$ be an element of $C_{0}^{*}(M)$. Then, $Q d \delta \xi=d \delta Q \xi$. Conversely, suppose $d \delta C_{0}^{*}(M)$ is an invariant subspace of $Q$. Then, there exists a $\xi \in C_{0}^{*}(M)$ such that $d \delta \xi$ is an eigenvector of $Q$, that is $Q d \delta \xi=(R / n) d \delta \xi \in C_{0}^{*}(M)$. Moreover, since $\Delta \delta \xi=(R /(n-1)) \delta \xi($ see $[1$, p. 264]),

$$
d \delta \xi=\frac{R}{n-1} \xi+\langle Y, \cdot\rangle
$$

where $Y$ is a Killing field. That this can only hold if $M$ has constant curvature is a consequence of the following:

LEMMA. Let $M$ be a compact Riemannian manifold on which there is a nonconstant function $\phi: M \rightarrow R$ whose gradient $\xi=d \phi \in C_{0}^{*}(M)$. Then, there are no nonzero tensors of the type $(r, s), 0<2(s-r) \leqq n$ invariant under $X$ where $\xi=\langle X, \cdot\rangle$.

The proof of this lemma is intended for a subsequent paper.

Setting

$$
T(A, B)=\tilde{R}(A, B)-\frac{R}{n}\langle A, B\rangle,
$$

where $\tilde{R}$ is the Ricci tensor, it can be shown that $\theta(\xi) T=0$. Since the Weyl conformal curvature tensor is invariant under $X$, we see by the lemma that $M$ is conformally flat. However, since $\theta(\xi) T$ vanishes, a further application of the lemma gives $T=0$, that is $M$ is an Einstein space. But a conformally flat Einstein space has constant curvature, and so by Proposition $4, M$ is globally isometric with a sphere. This proves Theorem 2 and generalizes Proposition 1.

\section{REFERENCES}

1. S. I. Goldberg, Curvature and homology, Academic Press, New York, 1962.

2. S. I. Goldberg and S. Kobayashi, The conformal transformation group of a compact homogeneous Riemannian manifold, Bull. Amer. Math. Soc. 68 (1962), 378-381.

3. A. Lichnerowicz, Sur les transformations conformes d'une variete riemannienne compacte, C. R. Acad. Sci. Paris 259 (1964), 697-700.

4. T. Nagano, The conformal transformations on a space with parallel Ricci tensor, J. Math. Soc. Japan 11 (1959), 10-14.

5. S. Sternberg, Lectures on differential geometry, Prentice-Hall, Englewood Cliffs, N. J., 1964.

6. K. Yano and T. Nagano, Einstein spaces admitting a one-parameter group of conformal transformations, Ann. of Math. 69 (1959), 451-461.

UNIVERSITY OF ILLINOIS 